\title{
A pilot randomized trial comparing long-term effects of red and white wines on biomarkers of atherosclerosis (In vino veritas: IVV trial)
}

\author{
Taborsky $\mathrm{M}^{1}$, Ostadal $\mathrm{P}^{2}$, Petrek $\mathrm{M}^{3}$ \\ First Clinic of Internal Medicine - Cardiology Clinic, Olomouc University Hospital, Olomouc, Czech Republic. \\ milos.taborsky@fnol.cz
}

\begin{abstract}
Background: Since early 90', growing body of evidence indicates that the Mediterranean diet with mild to moderate consumption of wine, mostly red wine, has a protective effect on cardiovascular diseases. Several mechanisms have been discussed to participate in the beneficial effect of red wine, such as antioxidant or vasodilating activity. However, later it has been shown that also other alcoholic beverages have a protective effect on atherosclerosis. Up to now, data from the prospective, long-term, head-to-head comparisons of the effects of different drinks on markers of atherosclerosis are insufficient.

Methods: The IVV (in vino veritas) study is a long-term, prospective, multicenter, randomized trial comparing the effect of red and white wines on the markers of atherosclerosis. One hundred and twenty healthy subjects with mild to moderate risk of atherosclerosis will be randomized to regular consumption of red wine (Pinot Noir) or white wine (Chardonnay-Pinot) for one year. The primary endpoint is the level of HDL-cholesterol at one year, while secondary endpoints are levels of other markers of atherosclerosis (LDL-cholesterol, C-reactive protein, myeloperoxidase, advanced oxidation protein product, interleukins 6 and 18, matrix metalloproteinases, glutathione s-transferase, monocyte chemoattractant protein 1 , soluble CD40L).

Conclusion: The IVV trial is the first study focusing on the long-term prospective comparison of the effects of red and white wines consumption on HDL-cholesterol and other markers of atherosclerosis. Results of the IVV trial may extend our understanding of the widely discussed "French paradox" (Tab. 1, Ref. 21). Full Text in PDF www.elis.sk.

Key words: red wine, white wine, atherosclerosis, HDL-cholesterol.
\end{abstract}

The observation of so-called "French paradox" by Renaud and Lorgeril in 1992 (1) started an enormous effort to discover the mechanisms of the beneficial effect of wine drinking on cardiovascular prognosis. Since early $90^{\prime}$ a number of experimental studies have been published, describing the protective effect of red wine on different pathways of the pathogenesis of atherosclerosis. Antioxidants flavonoids and polyphenols became the first substances contained in red wine with proven beneficial effect in various diseases, such as inhibition of LDL oxidation or attenuation of ischemia-reperfusion injury (2). However, several experimental studies have also shown that alcohol itself may have a protective impact (2) and these observations were confirmed by the data from large population-based studies and registries, showing cardiovascular benefits of alcohol drinking, regardless of type of beverage while similar benefits were shown for red and white wines, beer and even liquors $(3,4)$.

${ }^{1}$ First Clinic of Internal Medicine - Cardiology Clinic, Olomouc University Hospital, Olomouc, Czech Republic, ${ }^{2}$ Department of Cardiology, Na Homolce Hospital, Prague, Czech Republic, and ${ }^{3}$ Department of Biochemistry, Olomouc University Hospital, Olomouc, Czech Republic

Address for correspondence: M. Taborsky, MD, PhD, FESC, MBA, First Clinic of Internal Medicine - Cardiology Cloníc, Olomouc University Hospitál, I.P. Pavlova 6, CZ-779 00 Olomouc, Czech Republic.

Trial registration: ISRCTN54359610
It has been shown in animal experiments and in vitro studies that there are major differences in basic characteristics between red and white wines. Red wine exhibits higher antioxidant capacity and protective effect against LDL-oxidation as compared with white wine (5-7). Rosenkranz et al (8) and Sparwel et al (9) have shown attenuation of platelet-derived growth factor receptor (PDGFR) signaling (an important pathogenic mechanism in the development of atherosclerosis) in rat and human vascular smooth muscle cells (VSMCs) induced by red wine, whereas white wine had no effect. On the other hand, Munday et al (10) did not find any differences between red and white wine in serum lipoprotein profile and fatty streak formation in C57BL/6 mouse atherosclerosis model. López et al. (11) observed a similar reduction in superoxide production and an increase in expression and activity of NO-synthase (iNOS) by dealcoholized red and white wines in a rat model while up-regulation of cyclo-oxygenase-2 (COX-2) was detected only in the red wine group. Moreover, Auger et al (12) described that both red wine and polyphenols-enriched white wine prevent early atherosclerosis in hamsters.

Regarding the effect on atherosclerosis, there is only limited evidence from the prospective head-to-head comparisons of different alcohol beverages in human subjects; the published studies were focused mostly on acute effect of drinking. Tousoulis et al (13) observed reactive hyperemia after beer and red wine con- 
sumption that was not detected after white wine, whiskey or water in healthy young individuals. Reduction in urinary prostaglandin PGF2 $\alpha$-III as a marker of oxidative stress was more expressed after red wine than after white wine in a study on healthy subjects by Pignatelli et al (14). Pace-Asciak et al (15) did not find any advantage in red wine over white wine in preventing platelet aggregation in healthy males. Similar increase in total antioxidant capacity as a result of red or white wine consumption was described by Pinzani et al (16). Williams et al (17) did not observe any significant difference in acute effect of red and white wine drinking on blood pressure, heart rate, plasma lipids, levels of interleukin-6 (IL-6), intercellular adhesion molecule-1 (ICAM-1), and vascular cell adhesion molecule-1 (VCAM-1) in males with angiographic evidence of coronary artery disease (CAD). Moreover, comparable acute improvement of endothelial function after red and white wine drinking was reported by Whelan et al (18) in subjects with proven CAD. Blann et al (19) described increase in beta-thromboglobulin level after red wine whereas white wine had no effect. Until now, the longest exposition to red and white wines in a prospective trial was examined by Saranella et al (20) in their four-week study; they found in a group of thirty-six healthy females stronger anti-inflammatory effect of the red wine as compared with white wine consumption.

Hitherto, prospective data from the long-term, head-to-head comparisons of the effects of red and white wines on markers of atherosclerosis are missing (21).

\section{Methods}

\section{Objective}

The objective of the IVV trial is to compare the long-term effect of regular red and white wine drinking on the biomarkers of atherosclerosis.

\section{Study overview}

This is a prospective, multicenter, randomized, comparative study in one hundred and twenty individuals with mild to moderate risk of cardiovascular disease. Study protocol, information for patient, and informed consent are reviewed and approved by multicenter ethics committee as well as by local ethics committees in each institution before study initiation. Eligible individuals will be randomized to one regular drinking of either red wine (Pinot Noir, 2008, Moravia, Czech Republic) or white wine (Chardonnay-Pinot, 2008, Moravia, Czech Republic): women with body weight less than $70 \mathrm{~kg} 0.2$ liter per day, women over $70 \mathrm{~kg}$ and men 0.3 liter per day; chemical specifications of the respective wines are shown in Table 1. Participants will be followed on the intentionto-treat basis. Primary endpoint is the level of HDL-cholesterol, secondary endpoints are levels of other markers associated with progression of atherosclerosis (total- and LDL-cholesterol, triglycerides, oxidized LDL, C-reactive protein, advanced oxidation protein product, myeloperoxidase, interleukin 6 , interleukin 18 , matrix metalloproteinases, glutathione s-transferase, monocyte chemoattractant protein 1 , soluble CD40L, fatty acid binding protein).
Tab. 1. Chemical analysis of the selected white wine (ChardonnayPinot) and red wine (Pinot Noir).

\begin{tabular}{lcc}
\hline & Chardonnay-Pinot 2008 & Pinot Noir 2008 \\
\hline Polyphenols & 269 & 1974 \\
Flavanols & 11.9 & 607.4 \\
Antioxidant activity & 42.9 & 579.6 \\
Catechin & 4.07 & 143.81 \\
Epicatechin & 1.25 & 78.11 \\
Trans-resveratrol & 0.44 & 2.97 \\
Trans-piceid & 0.17 & 1.97 \\
Cis-resveratrol & 0.69 & 5.63 \\
Cis-piceid & 0.66 & 8.79 \\
Trans-piceatannol & 0.05 & 0.85 \\
Trans-astringin & 0.04 & 0.36 \\
Rutin & 0 & 3.37 \\
Myricetin & 0 & 0.55 \\
Quercetin & 0 & 0.10 \\
Tyrosol & 24.01 & 13.38 \\
Anthocyans & 0 & 71.20 \\
\hline
\end{tabular}

Data are expressed in $\mathrm{mg} / \mathrm{L}$. Both wines were grown and produced by Gala winery (Moravia, Czech Republic).

\section{Population}

One hundred and twenty asymptomatic individuals with mild to moderate risk of cardiovascular disease according to the HeartScore ${ }^{\circledR}(20)$ and without known acute or chronic inflammatory disease and liver or renal disease will be eligible for this study.

\section{Follow-up}

Eligible individuals willing to participate in the IVV study will be (after the signing of informed consent) randomized into one of the two arms. Red or white wine will be supplied in bottles directly to participants' home in the respective amount. Each participant also obtains a workbook with instruction to make notes about daily consumption of the study wine as well as other alcohol beverages. Blood samples for the measurement of laboratory endpoint and safety parameters will be obtained at a random visit and then at one, six, and twelve months. All visits include physical examination and assessment of cardiovascular events and other medical history since the last visit. At twelve months, after the final visit, the wine supply will be stopped.

During the study period no specific restrictions and recommendation are made with respect to diet, life-style or possible diagnostic or therapeutic procedures.

\section{Safety assessment}

Liver enzymes (alanine aminotransferase, aspartate aminotransferase, gamma glutamyltransferase, alkaline phosphatase) will be investigated at all visits. If any of the parameters exceed threefold $(>3 x)$ the upper limit of normal range (ULN), unscheduled safety visit will be performed in 7 to 14 days if any of the liver enzymes remains to be $>3 x$ ULN, wine consumption will be stopped.

\section{Sample size calculation}

Based on the data from short-term studies we expect a $13 \%$ increase in HDL-cholesterol in the red wine-group and a $5 \%$ rise in the white wine-group. With $\alpha=0.05$ and $80 \%$ power, the sample size to detect the significant difference in HDL-cholesterol was 
$156-158$

calculated as 51 participants per group. With the planned 60 participants per group the study will have an $86 \%$ power to detect the significant difference.

The IVV trial is the first study focusing on the long-term prospective comparison of the effect of red and white wine consumption on HDL-cholesterol and other markers of atherosclerosis. Results of the IVV trial may extend our understanding of the widely discussed "French paradox".

\section{References}

1. Renaud S, de Lorgeril M. Wine, alcohol, platelets, and the French paradox for coronary heart disease. Lancet 1992; 339 (8808): 1523-1526.

2. Das S, Santani DD, Dhalla NS. Experimental evidence for the cardioprotective effects of red wine. Exp Clin Cardiol 2007; 12 (1): 5-10.

3. Mukamal KJ, Conigrave KM, Mittleman MA, Camargo CA, Jr., Stampfer MJ, Willett WC et al. Roles of drinking pattern and type of alcohol consumed in coronary heart disease in men. N Engl J Med 2003; 348 (2): 109-118.

4. Samanek M, Dobiasova M, Urbanova Z, Kaspar Z, Omacka A. [Consumption of white wine retards development of atherosclerosis and affects anticoagulation]. Cas Lek Cesk 2002; 141 (8): 251-254.

5. Kalkan Yildirim H, Delen Akcay Y, Guvenc U, Yildirim Sozmen E. Protection capacity against low-density lipoprotein oxidation and antioxidant potential of some organic and non-organic wines. Int J Food Sci Nutr 2004; 55 (5): 351-362.

6. Jamroz A, Beltowski J. Antioxidant capacity of selected wines. Med Sci Monit 2001; 7 (6): 1198-1202.

7. Landrault N, Poucheret P, Ravel P, Gasc F, Cros G, Teissedre PL. Antioxidant capacities and phenolics levels of French wines from different varieties and vintages. J Agric Food Chem 2001; 49 (7): 3341-3348.

8. Rosenkranz S, Knirel D, Dietrich H, Flesch M, Erdmann E, Bohm M. Inhibition of the PDGF receptor by red wine flavonoids provides a molecular explanation for the "French paradox". FASEB J 2002; 16 (14): 1958-1960.

9. Sparwel J, Vantler M, Caglayan E, Kappert K, Fries JW, Dietrich $\mathbf{H}$, et al. Differential effects of red and white wines on inhibition of the platelet-derived growth factor receptor: impact of the mash fermentation. Cardiovasc Res 2009; 81 (4): 758-770.

10. Munday JS, Thompson KG, James KA, Manktelow BW. The effect of moderate alcohol consumption as either red or white wine in the C57BL/6 mouse atherosclerosis model. Coron Artery Dis 1999; 10 (2): 97-102.
11. Lopez D, Pavelkova M, Gallova L, Simonetti P, Gardana C, Lojek A, et al. Dealcoholized red and white wines decrease oxidative stress associated with inflammation in rats. Br J Nutr 2007; 98 (3): 611-619.

12. Auger C, Rouanet JM, Vanderlinde R, Bornet A, Decorde K, Lequeux N, et al. Polyphenols-enriched Chardonnay white wine and sparkling Pinot Noir red wine identically prevent early atherosclerosis in hamsters. J Agric Food Chem 2005; 53 (25): 9823-9829.

13. Tousoulis D, Ntarladimas I, Antoniades C, Vasiliadou C, Tentolouris $\mathbf{C}$, Papageorgiou $\mathbf{N}$, et al. Acute effects of different alcoholic beverages on vascular endothelium, inflammatory markers and thrombosis fibrinolysis system. Clin Nutr 2008; 27 (4): 594-600.

14. Pignatelli P, Ghiselli A, Buchetti B, Carnevale R, Natella F, Germano G, et al. Polyphenols synergistically inhibit oxidative stress in subjects given red and white wine. Atherosclerosis 2006; 188 (1): 77-83.

15. Pace-Asciak CR, Rounova O, Hahn SE, Diamandis EP, Goldberg DM. Wines and grape juices as modulators of platelet aggregation in healthy human subjects. Clin Chim Acta 1996; 246 (1-2): 163-182.

16. Pinzani P, Petruzzi E, Magnolfi SU, Malentacchi F, De Siena G, Petruzzi I, et al. Red or white wine assumption and serum antioxidant capacity. Arch Gerontol Geriatr 2010.

17. Williams MJ, Sutherland WH, Whelan AP, McCormick MP, de Jong SA. Acute effect of drinking red and white wines on circulating levels of inflammation-sensitive molecules in men with coronary artery disease. Metabolism 2004; 53 (3): 318-323.

18. Whelan AP, Sutherland WH, McCormick MP, Yeoman DJ, de Jong SA, Williams MJ. Effects of white and red wine on endothelial function in subjects with coronary artery disease. Intern Med J 2004; 34 (5): 224-822.

19. Blann AD, Williams NR, Lip GY, Rajput-Williams J, Howard AN. Acute ingestion of red wine by men activates platelets but does not influence endothelial markers: no effect of white wine. Blood Coagul Fibrinolysis 2002; 13 (7): 647-651.

20. Sacanella E, Vazquez-Agell M, Mena MP, Antunez E, FernandezSola J, Nicolas JM, et al. Down-regulation of adhesion molecules and other inflammatory biomarkers after moderate wine consumption in healthy women: a randomized trial. Am J Clin Nutr 2007; 86 (5): 1463-1469.

21. Ronksley PE, Brien SE, Turner B, et al. Association of alcohol consumption with selected cardiovascular disease outcomes: a systematic review and meta-analysis. BMJ 2011: 342; 671. doi: 10.1136/bmj.d671.

Received March 8, 2011. Accepted January 9, 2012. 